\title{
Fighting fat
}

\author{
New drugs against obesity in the pipeline
}

A growing epidemic is sweeping through the developed world, threatening millions with disability and death: obesity. 'Epidemic' may sound exaggerated, but the facts speak for themselves: 154 million people worldwide are obese-or more than $20 \%$ are over their ideal body weight-including more than $50 \%$ of all Americans. More disturbing is the prevalence of childhood obesity, which has jumped dramatically over the past 20 years and now accounts for a doubling in the incidence of diabetes, a 5-fold increase in sleep apnoea and a 3 -fold increase in gall bladder disease. The World Health Organization and the US Surgeon General have already warned that obesity is a serious, life-threatening disease. Indeed, as a major risk factor for hypertension, stroke, heart disease, diabetes and possibly certain forms of cancer, obesity exacts a greater toll on health and healthcare costs than either smoking or drinking. In the USA alone, the direct medical costs of obesity-related diseases account for $6 \%$ of the nation's entire healthcare budget. Obesity is, in short, a great deal more than a 'lifestyle' or cosmetic problem.

This has not been lost on the pharmaceutical industry. Companies know that the worldwide market for an effective treatment for obesity is gargantuan: analysts estimate that an effective and safe drug could generate as much as US\$26 billion per year in the USA alone. 'It is probably the largest pharmaceutical market ever, and carries with it 27-30 dangerous co-morbidities,' said Louis Tartaglia, Vice President of metabolic diseases at Millennium Pharmaceuticals (Cambridge, MA), who estimates the current annual worldwide market conservatively at US\$10-15 billion. If one adds the patients who are obese and diabetic, the number soars even higher, as obesity and diabetes are linked diseases.

The sudden rise in obesity is mainly a result of lifestyle changes, particularly the ready availability of calorie-laden, refined food and a decrease in physical activity. 'Genes have not changed in the past
50 years, but our eating and exercising habits have,' said George Yancopoulos, Chief Scientific Officer and President of Regeneron Pharmaceuticals (Tarrytown,

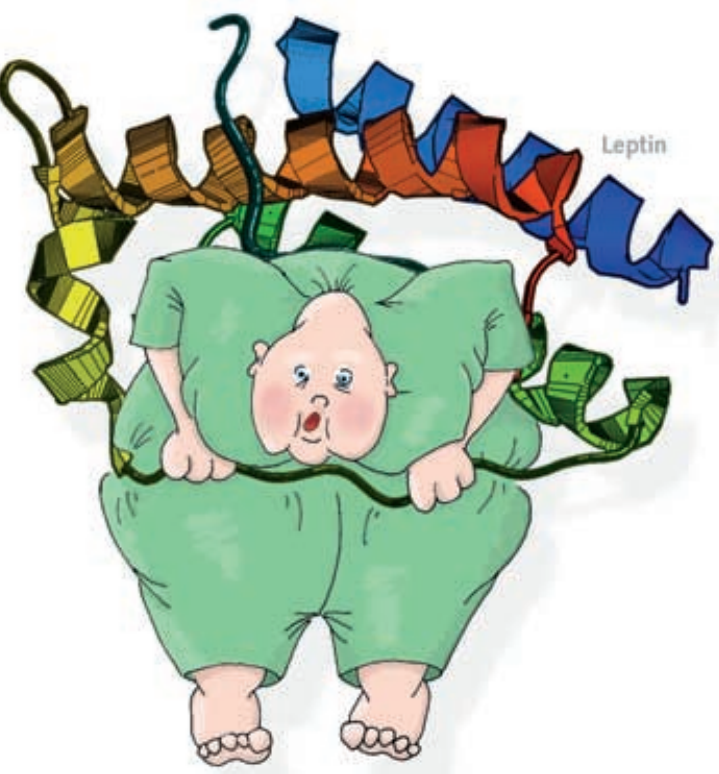

NY), one of many companies working on drugs to treat obesity. But it cannot be lifestyle alone. Certain ethnic groups have a higher prevalence of obesity, and the discovery of a number of genes that regulate fat storage point to an interaction between genetic and environmental factors.

Indeed, research on fat metabolism has made large strides forward over the last decade and has challenged some established views. 'One of the most important advances in obesity research is the realisation that fat is an endocrine tissue,'

\section{Companies know that the market \\ for an effective treatment for obesity is gargantuan}

said Barbara Kahn, Professor of Endocrinology at Harvard University's School of Medicine. Another step forward was the discovery of a number of peptides that function as central regulators of food intake and energy homeostasis. These findings point to a complex involvement of the neuroendocrine system, where the central nervous system and fat interact through hormones and neurotransmitters, and affect other organs, most notably the liver and the pancreas.

Given the complexity of this system, it is no surprise that most treatments have been relatively ineffective or fraught with serious health risks, such as addiction to amphetamine diet pills, heart valve damage and hypertension. Furthermore, only a few drugs have reached the market recently. Xenical (Orlistat), a pancreatic lipase inhibitor developed by HoffmannLaRoche (Geneva, Switzerland), works by blocking the absorption of fat in the intestine, but can cause uncomfortable side-effects and has been linked to an increased incidence of cancer. Meridia (known as Reductil in the UK), originally developed by Knoll AG (Ludwidgshafen, Germany), is a serotonin and norepinephrine reuptake inhibitor that is thought to act on the appetite centre of the hypothalamus. However, it often causes elevated blood pressure, and its producer, Abbott Labs (Abbott Park, IL) admitted in March that 34 patients in the USA have died after taking the drug. Italy suspended sales of the drug after receiving 50 reports of adverse reactions, including two deaths. The anti-depressant Wellbutrin (GlaxoWellcome, Research Triangle Park, $\mathrm{NC}$ ) acts in the same way as Meridia but has only a very mild weight-reduction effect.

But recent findings on fat metabolism open new avenues for developing effective drugs. 'Body weight is regulated through a series of feedback systems that provide targets for effective drug treatment and strategies for drug development,' Peter Kopelman from Queen Mary's School of Medicine \& Dentistry at the University of London (UK) said. Food intake can be reduced either by amplifying anorexigenic (anti-appetite) signals or blocking orexigenic (pro-appetite) factors. Other strategies aim at increasing energy expenditure by uncoupling fuel metabolism from the generation of ATP. It is also possible to modulate fat metabolism by 
regulating fat synthesis or lipolysis or by moderating the fixed 'internal reference point' maintained by the central controller of body weight. An effective anti-obesity treatment must target a number of these regulatory points. 'New drugs must ultimately affect both energy intake and energy expenditure, to have a significant and long-lasting impact on body weight,' according to Kopelman. 'Drugs that work purely on one side of the energy balancethat is, energy intake or expenditure-are unlikely to achieve long-term efficacy because of compensatory adjustments ensuring the maintenance of body fat homeostasis.'

Thus, the major leap in the development of more effective drugs has come through a better understanding of fat metabolism. In particular, the discovery and cloning of the adipocyte-derived hormone leptin and its receptor proved to be major breakthroughs. Leptin is essential for normal body weight regulation and scientists had thought that the obese need leptin in a similar way to diabetic patients who need insulin to process glucose. However, they soon discovered that like most diabetics who are actually insulin-resistant, most obese patients produce plenty of endogenous leptin, but are, in fact, leptinresistant. Recent research has also begun to uncover the mechanisms of insulinand leptin-resistance, and the link between obesity and diabetes.

And the pipeline for new drugs is bulging. The most promising, now in phase III clinical trials, is Regeneron's Axokine, an engineered version of ciliary neurotrophic factor, originally developed to treat amyotrophic lateral sclerosis (ALS). Serendipitously, Regeneron discovered that it led to unexpected and substantial weight loss in patients. 'The ALS patients given the factor were not getting weaker because they were losing fat, not muscle,' Yancopoulos said. In April 2002, his group demonstrated in mice that the drug reduces fat by activating the leptin pathway and does not cause rebound weight gain even in a leptin-resistant model of obesity.

Axokine mimics the ability of leptin to generate fat loss by enabling the patient to be satisfied with less food. The problem with dieting is that the body compensates by slowing down its metabolism and sending strong messages to the dieter to eat; the weight is regained because the body preserves its fat reserves and tells one to eat more, Yancopolous noted. Axokine sidesteps these mechanisms. It also works in diet-induced obesity, where there is sufficient leptin but resistance to the hormone-this represents the lion's share of cases of human obesity. 'Leptin was only intended to act as a satiety signal, not as a means to deal with too much body fat. Axokine works by superactivating the leptin pathway, including 5-10 neuropeptides, and turning on the satiety signal,' Yancopoulos said.

But there is more to leptin than just inducing satiety. Harvard's Kahn demonstrated that it acts on another component of the metabolism. 'Leptin stimulates the oxidation of fatty acids and the uptake of

\section{An effective obesity drug must target a number of regulatory points}

glucose, and prevents the accumulation of lipids in non-adipose tissues,' she said. Specifically, leptin inhibits the activity of acetyl co-enzyme A carboxylase (ACC), which stimulates the oxidation of fatty acids in skeletal muscle. 'ACC provides a pivotal step in fuel metabolism as it links fatty-acid and carbohydrate metabolism through the shared intermediate acetyl co-enzyme A,' the researchers wrote. Kahn thus thinks that the ACC pathway might provide new drug targets.

Millennium is working on a small molecule that will move more freely across the blood-brain barrier than leptin, and which super-stimulates the leptin receptor. It is also examining the littleunderstood downstream targets in the leptin-signalling pathway, which could help prevent leptin resistance. 'It is a hotly debated subject which is the best target,' Tartaglia said. Discovering which proteins generate resistance to leptin and which activate the receptor could, taken together, prove to be the 'magic bullet' for obesity, he thinks.

Biovitrum in Stockholm, Sweden, is also aiming at the neurological component of obesity and testing a selective serotonin reuptake inhibitor, BVT.933, in phase II clinical trials. 'Rather than hitting on all 14 human serotonin receptors by increasing the serotonin levels, only one, the 2C receptor, gets activated by BVT.933,' Johan Kordel, Senior Vice President of research, explained. Patients treated with BVT.933 achieved a statistically significant and clinically relevant weight reduction compared with placebo.

Recent research points to another interesting target besides leptin-PTP1B, a protein-tyrosine phosphatase. Barbara Kahn and her colleague Benjamin Neel, from Harvard Medical School together with Millennium's Tartaglia showed that PTP1B regulates leptin signal transduction. Transgenic, PTP1B-deficient mice have decreased leptin/body fat ratios and leptin hypersensitivity; in short, these mice were insulin-sensitive and lean. 'We also found that lowered levels of PTP1B increased energy expenditure, and conversely, elevated levels of PTP1B in both muscle and fat, which contributes to insulin resistance,' Kahn said, calling PTP1B 'an outstanding drug target'. Michel Tremblay of the Department of Biochemistry at McGill University in Montreal, Canada, who is also working on PTP1B, estimated that at least 20 companies are now pursuing this drug target, including Merck (Rahway, NJ), NovoNordisk (Bagsværd, Denmark), Ely Lilly (Indianapolis, IN) together with Ceptyr, a new Seattlebased company focusing on phosphatases, Akros (Princeton, NJ), the US arm of Japan Tobacco and Pharmacia (Peapack, NJ).

The problem has been in designing a molecule that specifically inhibits PTP1B. Isis Pharmaceuticals (Carlsbad, CA) is confident that it has found one. Brett Monia, Isis's Vice President of antisense drug discovery and his colleagues have developed an antisense nucleotide to selectively block PTP1B gene expression. A year ago, it licensed the drug, Isis113715 to Merck. The group also published a proof of principle paper in April 2002, in which they demonstrated that the antisense construct inhibited PTEN, another lipid phosphatase that is involved in the insulin signalling pathway. The antisense oligonucleotide normalised blood glucose concentrations in diabetic and obese mice and lowered serum triglyceride and cholesterol concentrations. 'Antisense technology has an advantage of being able to block the production of a protein, in this case, PTP1B, rather than just inhibiting it once it is produced,' Frank Bennett, Isis's Vice President of antisense research, said. To date, no adverse effects have been seen with the use of Isis's antisense drugs in small and large animal models of diabetes and obesity, he added. 


\section{analysis}

Another target receiving serious attention is the enzyme fatty acid synthase. Daniel Lane from Johns Hopkins University in Baltimore, MD, and colleagues have shown that a specific inhibitor, dubbed $\mathrm{C} 75$, reduces both food intake and body weight in mice, and interrupts fastinginduced hunger signals in lean mice. In obese mice, C75 suppresses food intake, reduces body weight, and normalises obesity-associated high blood sugar and high insulin levels. Johns Hopkins University, which holds the patent to $\mathrm{C} 75$, has licensed it to FASgen, a new company in Baltimore.

Finally, older substances may yield new drugs as well: a small region of the human growth hormone appears to have a specific effect on fat without any effect on either growth or insulin resistance, according to Frank $\mathrm{Ng}$ of Monash University (Melbourne, Australia). With the support

The major leap in the development of new drugs has come through a better understanding of fat metabolism

from Metabolic Pharmaceuticals, Ng has developed it into an orally administered drug, which is now in phase lla testing.

Basic research to uncover fat metabolism and applied research to find effective treatments against obesity have received a lot of attention over the last years both in terms of media coverage and funding. And even if the interest in developing effective treatments against being overweight has a strong lifestyle component, it is increasingly crucial to public health. New treatments are needed to help those millions of people suffering from obesityespecially the dramatically rising number of overweight children who are at risk of lifelong diabetes and the accompanying risk of heart disease and disability.

\section{Vicki Brower}

DOI: 10.1093/embo-reports/kvf148 